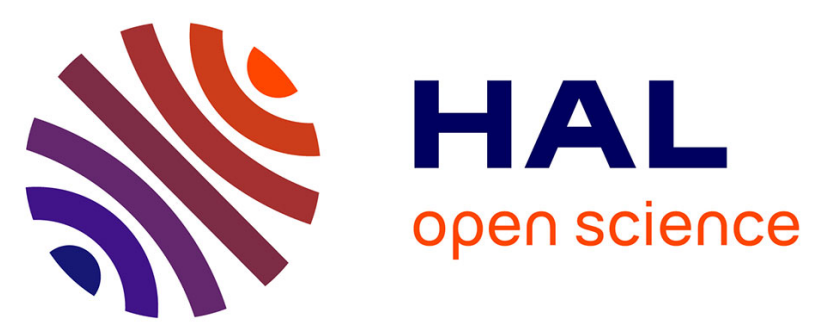

\title{
Terahertz spintronic emitter with arbitrarily controllable linear polarization properties not needing a rotating magnetic bias
}

Pierre Kolejak, Kamil Postava, Jean-Francois Lampin, Nicolas Tiercelin, Mathias Vanwolleghem

\section{To cite this version:}

Pierre Kolejak, Kamil Postava, Jean-Francois Lampin, Nicolas Tiercelin, Mathias Vanwolleghem. Terahertz spintronic emitter with arbitrarily controllable linear polarization properties not needing a rotating magnetic bias. 45th International Conference on Infrared, Millimeter, and Terahertz Waves (IRMMW-THz), Nov 2020, Buffalo, NY (Virtual), United States. pp.1-2, 10.1109/IRMMWTHz46771.2020.9370505 . hal-03322811

\section{HAL Id: hal-03322811 \\ https://hal.science/hal-03322811}

Submitted on 30 Aug 2021

HAL is a multi-disciplinary open access archive for the deposit and dissemination of scientific research documents, whether they are published or not. The documents may come from teaching and research institutions in France or abroad, or from public or private research centers.
L'archive ouverte pluridisciplinaire HAL, est destinée au dépôt et à la diffusion de documents scientifiques de niveau recherche, publiés ou non, émanant des établissements d'enseignement et de recherche français ou étrangers, des laboratoires publics ou privés. 


\title{
Terahertz spintronic emitter with arbitrarily controllable linear polarization properties not needing a rotating magnetic bias
}

\author{
Pierre Koleják ${ }^{1,2}$, Kamil Postava ${ }^{1}$, Jean-François Lampin ${ }^{2}$, Nicolas Tiercelin ${ }^{2}$ and Mathias Vanwolleghem² \\ ${ }^{1}$ VSB-Technical University of Ostrava, Nanotechnology centre and IT4Innovations, 17. listopadu 15, 70833 \\ Ostrava-Poruba, Czech Republic \\ ${ }^{2}$ Institut d'Electronique, de Microelectronique et de Nanotechnologie, CNRS, 59652 Villeneuve d'Ascq, France
}

\begin{abstract}
Spintronic THz emitters are new intensively studied sources of $\mathrm{THz}$ radiation with high potential in broadband THz time-domain spectroscopy. We present a novel architecture for a spintronic emitter based on a ferromagnetic heterostructure with an induced in-plane magnetic anisotropy. This new approach allows to generate an arbitrary linear $\mathrm{THz}$ polarization taking advantage of the coherent Stoner-Wohlfarth rotation of the magnetic heterostructure. As a result the linear $\mathrm{THz}$ polarization state can be tuned without rotating the applied magnetic field. Combined with the huge bandwidth of the emitter, this may lead to high impact for terahertz ellipsometry and polarimetry.
\end{abstract}

\section{INTRODUCTION}

Present day $\mathrm{THz}$ time-domain spectroscopy methods are powerful phase sensitive tools with wide ranging applications from biology to solid-state physics. The majority of TDS sources and detectors are significantly limited by relaxation times and phonon absorptions. Typical TDS spectra are cut-off maximally at $6 \mathrm{THz}$. THz spintronic emitters, introduced 5 years ago by Kampfrath et al. [1,2], allow a new approach to break this limit. Exciting a $5 \mathrm{~d} / 3 \mathrm{~d}$ nonmagnetic (NM)/ferromagnetic (FM) multilayer by IR fs- pulses generates a spin-polarized current that upon diffusion in the NM metal undergoes strong spin-orbit coupling. The action of the inverse spin-Hall effect transforms the spin-current into a charge current dipole transversely oriented to the spin direction. The ultrafast relaxation times and absence of phonon absorption bands typical of metals allow the generation of THz pulses Fourier-limited by the IR pulse. Gapless spectra up to $30 \mathrm{THz}$ have been reported. The polarization state is intrinsically linked to the magnetization direction in the FM layer. While optimizations of the $\mathrm{THz}$ power have been reported, little work has been done on the control of the $\mathrm{THz}$ polarization state. We demonstrate a new approach of coherent control of the magnetization state of a spintronic emitter and thereby a controlled generation of an arbitrary linear $\mathrm{THz}$ polarization.

\section{RESULTS}

The THz polarization state is related to the magnetic properties of the spintronic emitter. The single FM layer is replaced by a FM heterostructure, consisting of several $\mathrm{FeCo} / \mathrm{TbCo}$ bilayers, to induce strong uniaxial magnetic anisotropy. This is the result of the magnetic exchange coupling of hard ( $\mathrm{FeCo}$ ) and soft $(\mathrm{TbCo})$ magnetic material in periodic stacking. The spintronic emitter is investigated in a reflection configuration with $100 \mathrm{fs}$ infrared pulse (at $820 \mathrm{~nm}$ ) incident under $45^{\circ}$. 2,5 periods of $10 \AA / 9 \AA$ $\mathrm{FeCo} / \mathrm{TbCo}$, sandwiched between $3 \mathrm{~nm} \mathrm{~W}$ and $110 \mathrm{~nm}$ Pt as NM layers, are sputtered deposited on $\mathrm{Si}$ substrate. The emitter is placed in a homogeneous magnetic field controlled by an in-house built small electromagnet $(50 \mathrm{mT})$. The strong uniaxial magnetic anisotropy leads to an in-plane coherent StonerWohlfarth rotation of the magnetization by varying the magnetic bias solely along the hard axis of the magnetic heterostructure. As the THz polarization is perpendicular to $\vec{M}$, coherent rotation of $\vec{M}$ tunes the $\mathrm{THz}$ polarization to an arbitrary linear state. The longitudinal and transverse component of the emission is measured by combining a polarization rotator with a dipole photoconductive antenna. Fig. 1 plots the peak of the measured temporal $\mathrm{THz}$ signal as a function of applied magnetic bias along the hard axis. The superposition of both field components (blue) describes the magnetic dependence of the polarization profile. As can be seen from the near-circular projection on a plane (green), varying solely 
the hard axis applied magnetic bias allows to obtain any linear polarization state with approximately the same field strength.

This $\mathrm{THz}$ vectorial measurement reproduces perfectly the vibrating sample magnetometer (VSM) hysteresis loops as is demonstrated in Fig. 2. The hysteresis loops are measured on the maximal peak position of terahertz waveforms using THz- TDS as well as results presented in Fig. 1. Besides hysteresis loops measured along the hard axis for both longitudinal (blue) and transverse (red) polarization components, the longitudinal component for the magnetic field applied along the easy axis (green) was also measured. Obtained hysteresis loops verify the strong uniaxial magnetic anisotropy induced by the ferromagnetic $\mathrm{FeCo} / \mathrm{TbCo}$ heterostructure. Moreover, measured THz-TDS hysteresis loops (full line) compared with VSM hysteresis loops (dashed) show the direct dependence of the generated terahertz polarization states on the in-plane magnetic properties of the spintronic emitter.

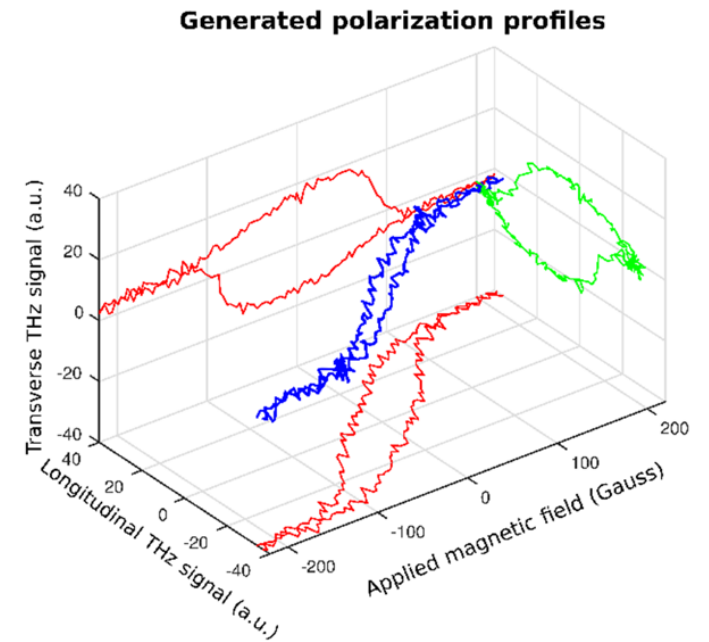

Fig.1 Polarization control in a unidirectional magnetic field. Field components (red) reflect the magnetic hysteresis. Their superposition (blue) and its projection (green) on the xy-plane gives the polarization trace during the loop.

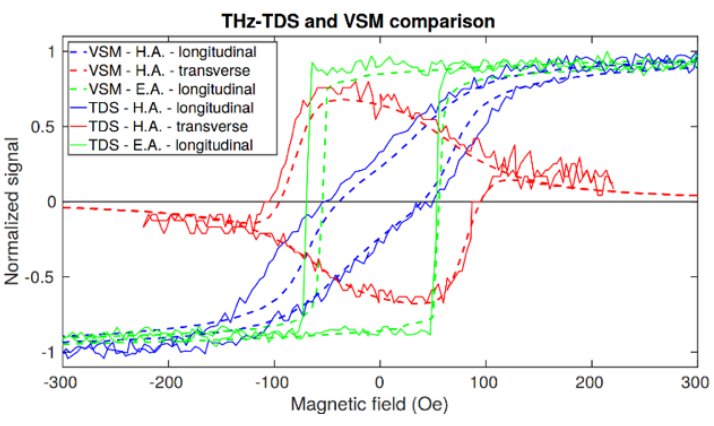

Fig.2 Comparison of magnetic hysteresis loops obtained using THz-TDS measurement (full line) on the maximal peak position with standard VSM measurements (dashed). Hysteresis loops applied along the hard axis were measured for both longitudinal (blue) and transverse (red) components. The hysteresis loop with the field applied along the easy axis for the longitudinal component (green) is also presented.

\section{SUMMARY}

We propose a new architecture for a spintronic TDS emitter based on a ferromagnetic heterostructure with an induced uniaxial magnetic anisotropy. The emitter generates an arbitrary linear polarization of the THz pulse using coherent rotation of the magnetization. We show the direct dependence between the terahertz linear polarization components on the in- plane magnetic properties of the spintronic emitter. This opens up possibilities for $\mathrm{THz}$ ellipsometry without any moving or rotating parts.

\section{REFERENCES}

[1]. T. Kampfrath, et al., "Terahertz spin current pulses controlled by magnetic heterostructures," Nat. Nanotechnol., vol. 8, pp. 256, 2013.

[2]. T. Seifert, et al., "Efficient metallic spintronic emitters of ultrabroadband terahertz radiation," Nat. Photonics., vol. 10, pp. 483, 2016. 